\title{
Nondestructive Quantification of Heavy Elements Through the Analysis of Beam Hardening Artifacts Using Double-Exposure X-ray Computed Tomography: A Theoretical Consideration
}

\author{
Yoshito Nakashima' ${ }^{1}$. Tsukasa Nakano' ${ }^{1}$ \\ Received: 26 November 2019 / Accepted: 21 March 2020 / Published online: 29 March 2020 \\ (c) The Author(s) 2020
}

\begin{abstract}
We present a theoretical background for heavy element quantification through the intensive analysis of beam hardening (cupping artifacts) in X-ray computed tomography (CT) images. Cupping artifacts resulting from X-ray CT using a polychromatic X-ray source are quantitatively analyzed with an analytical solution for a cylindrical sample of a homogeneous aqueous solution/suspension containing a heavy element. The theoretical solution reveals that the severity of cupping artifacts is strongly dependent on the sample chemistry and the acceleration voltage of the X-ray tube. Careful analysis of this dependency enabled simultaneous determination of the atomic number and molar concentration of the heavy element within a particular estimation error range. Significant improvement in terms of the accuracy of determining the atomic number was achieved by employing double-exposure X-ray CT.
\end{abstract}

Keywords Beam hardening · Double-exposure X-ray computed tomography $\cdot$ Heavy-element quantification $\cdot$ Image analysis $\cdot$ Nondestructive measurement

\section{Introduction}

Quantification of heavy elements in samples is one of the most important subjects in analytical chemistry [1]. Substances that contain heavy elements are often toxic and hazardous; therefore, nondestructive methods to safely analyze such heavy elements confined in containers are desirable, such as in cargo security [2] and in analysis of polluted wastes, water and soils [3-6]. The X-ray computed tomography $(\mathrm{CT})$ is one of useful methods for the nondestructive discrimination of heavy elements in samples [7]. However, heavy elements yield undesirable beam hardening artifacts (cupping artifacts) in the reconstructed CT images due to the polychromatic X-ray source [8]. This cupping artifact occurs as a characteristic cupped shape for the profile of the voxel values across a homogeneous sample. The cupping artifact yielding the inhomogeneous profiles even for homogeneous

Yoshito Nakashima

nakashima.yoshito@aist.go.jp

1 National Institute of Advanced Industrial Science and Technology (AIST), Central 7, Higashi 1-1-1, Tsukuba, Ibaraki 305-8567, Japan samples could reduce the accuracy of heavy element discrimination when the conventional X-ray CT method is employed. Nakashima and Nakano $[9,10]$ proposed a novel image analysis method using single-exposure X-ray CT (i.e., using a single acceleration voltage for the $\mathrm{X}$-ray tube). The method enables simultaneous estimation of the atomic number and concentration of a heavy element confined in a sample by carefully analyzing the cupping profiles. This method $[9,10]$ can be implemented using conventional CT systems without energy-resolved [11, 12] special detectors.

However, the constraints used for the material differentiation provided by the single-exposure CT method were not complete. As a result, this method failed to eliminate heavyelement candidates with abnormally high atomic numbers (e.g., ${ }_{75} \mathrm{Re}$ and ${ }_{76} \mathrm{Os}$ ) relative to the ground truth (e.g., ${ }_{58} \mathrm{Ce}$ ) [9]. In order to eliminate such candidates, we propose the use of double-exposure X-ray CT (i.e., acquisition of two CT images using two different acceleration voltages) that provides more constraints on the differentiation of heavy elements in samples. The validity of the proposed method was evaluated theoretically by analyzing an exact solution of the cupping artifact for a homogeneous cylindrical sample of an aqueous solution/suspension containing a heavy element. 


\section{Methods}

\subsection{Principle of Heavy Element Quantification Using Beam Hardening}

In this section, we describe the principle for determining the concentration and atomic number of a heavy element in a sample through quantitative analysis of an X-ray CT image containing a beam hardening artifact. Consider a cylindrical homogeneous sample of $43.0 \mathrm{mM}$ of an iodine aqueous solution (4-cm radius). The linear attenuation coefficient (LAC) spectrum $\mu$ of the sample shows a strong dependence on the X-ray photon energy $E$. This phenomenon is largely caused by the heavy element iodine, which is a strong X-ray absorber (Fig. 1). When this sample is imaged by an X-ray CT system using X-rays accelerated at $100 \mathrm{kV}$ (Fig. 1), a beam hardening artifact (i.e., cupping artifact) appears because of the polychromaticity of the X-ray source spectrum. The cupping artifact in the reconstructed CT image of the homogeneous sample is characterized by a large LAC value near the sample rim and small LAC value at the sample center (Fig. 2a) [13-17]. The reconstructed LAC value

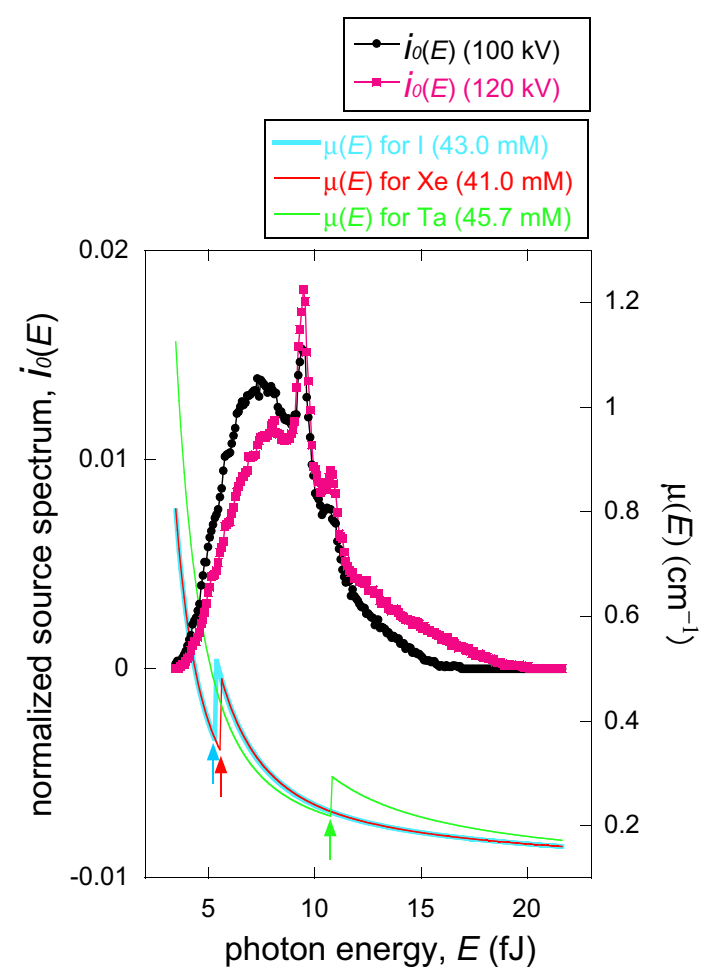

Fig. 1 Representative examples of the photon-energy spectra for an $\mathrm{X}$-ray source accelerated at 100 and $120 \mathrm{kV}$ [14]. Each sum is normalized for unification. The LAC spectra $\mu(E)$ for $\mathrm{I}_{-} \mathrm{H}_{2} \mathrm{O}, \mathrm{Xe}-\mathrm{H}_{2} \mathrm{O}$, and $\mathrm{Ta}-\mathrm{H}_{2} \mathrm{O}$ systems with specific molar concentrations are superimposed. The $\mathrm{K}$ absorption edges are indicated by arrows $(5.3,5.5$, and $10.8 \mathrm{fJ}$ for ${ }_{53} \mathrm{I},{ }_{54} \mathrm{Xe}$, and ${ }_{73} \mathrm{Ta}$, respectively) distribution in the radial direction (Fig. 2b) is a consequence of the physical interaction of (i) the polychromatic source spectrum $i_{0}(E)$ of the X-rays irradiated from the tube and (ii) LAC spectrum $\mu(E)$ derived from the sample chemistry. Therefore, if the X-ray source spectrum $i_{0}(E)$ is known, it is possible to estimate the sample chemistry (the atomic number and molar concentration of the heavy element) by carefully analyzing the cupping profile in Fig. $2 \mathrm{~b}$. This is the main principle of heavy element quantification based on $\mathrm{CT}$ image analysis $[9,10]$.

This principle has been applied to a CT image of a 550$\mathrm{mM} \mathrm{CeCl} \mathrm{C}_{3}$ aqueous solution in a container [9]. The CT image was obtained experimentally by using a single acceleration voltage of $100 \mathrm{kV}$. Through numerical simulations of CT image reconstruction obtained by considering various heavy elements for the container image, a trial-and-error search was performed for the atomic numbers of the heavy element candidates that most reasonably reproduced the experimentally obtained cupping profile. This search for appropriate atomic numbers was largely successful. However, analysis using the single-exposure CT image method failed to eliminate heavy element candidates with abnormally high atomic numbers (i.e., ${ }_{75} \mathrm{Re}$ and ${ }_{76} \mathrm{Os}$ ) relative to the ground truth (i.e., ${ }_{58} \mathrm{Ce}$ ) [9]. This is because the cupping profiles for ${ }_{75} \mathrm{Re}$ and ${ }_{76} \mathrm{Os}$ are nearly the same as that for ${ }_{58} \mathrm{Ce}$ when a CT image is acquired at $100 \mathrm{kV}$.

In this paper, we propose an improved method to eliminate such candidates through the use of double-exposure CT. This method requires the acquisition of two CT images using two different acceleration voltages. The cupping profile (e.g., Fig. 2b) is dependent on the X-ray source spectrum, as well as the sample chemistry. Therefore, CT images obtained with different acceleration voltages for the $\mathrm{X}$-ray tube provide different constraints that are useful for the identification of an unknown heavy element in a sample. The verification of this concept based on theoretical calculations is presented in the following section.

\subsection{Heavy Element Quantification Using Double-Exposure CT}

In this section, we discuss the determination of the atomic number and concentration of a heavy element in a homogeneous cylindrical sample using double-exposure CT. Determination is performed by systematically calculating the effects of sample chemistry and acceleration voltage on the cupping profile. Cupping profiles that are numerically obtained using image-reconstruction computer software [9, $13,17]$ inevitably contain errors that are introduced by the discretization of sample shapes $[13,18]$ (see the noisy fluctuation of the profile in Fig. 2b). Therefore, an analytical solution [19] was employed for cupping profile analysis in this study to avoid such undesirable errors. 


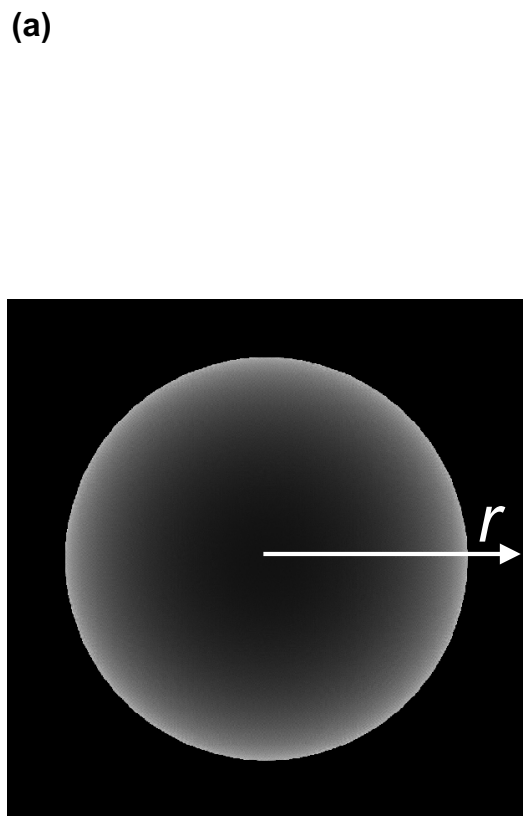

Fig. 2 Example cupping artifact obtained via calculation. a Numerically reconstructed $[9,13,19]$ two-dimensional CT image of a cylindrical sample of the homogeneous $\mathrm{I}_{-} \mathrm{H}_{2} \mathrm{O}$ mixture $(43.0 \mathrm{mM}$ of iodine). A Ramachandran reconstruction filter with a finite Nyquist wavenumber of $50 \mathrm{~cm}^{-1}$ was used. The total image size is $1024^{2}$ voxels and the radius $R$ of the cylindrical sample is 400 voxels $(4 \mathrm{~cm})$. The $r$ axis increases from the sample center to the image edge. b Distribution of the voxel values, $f(r)$, along the $r$ axis for the image in a. A cupping artifact (i.e., decreasing voxel value toward the sam-

The theoretical cupping profile $f(r)$ for a homogeneous cylindrical sample is given as

$f(r)=\sum_{n=1}^{N} \frac{2^{n}}{\sqrt{\pi}} \frac{\Gamma\left(\frac{n}{2}+1\right)}{\Gamma\left(\frac{n+1}{2}\right)} C_{n}\left(R^{2}-r^{2}\right)^{\frac{n-1}{2}}$

for $0 \leq r<R$, where $R$ is the sample radius, $r$ is the coordinate in the radial direction (Fig. 2a), $N$ is an integer that represents the upper limit of the summation, and $\Gamma$ is the gamma function [19]. No bowtie filter [20] for beam hardening reduction is assumed. The coefficient $C_{n}$ can be calculated explicitly based on the X-ray source spectrum and LAC spectrum of the sample. For example, the coefficient $C_{1}$ is

$C_{1}=\int_{0}^{E_{\max }} i_{0}(E) \mu(E) d E$,

where $i_{0}(E)$ is the normalized X-ray source spectrum [19], and $E_{\max }$ is the maximum value of the X-ray photon energy. Theoretically $E_{\max }$ is determined by the acceleration voltage

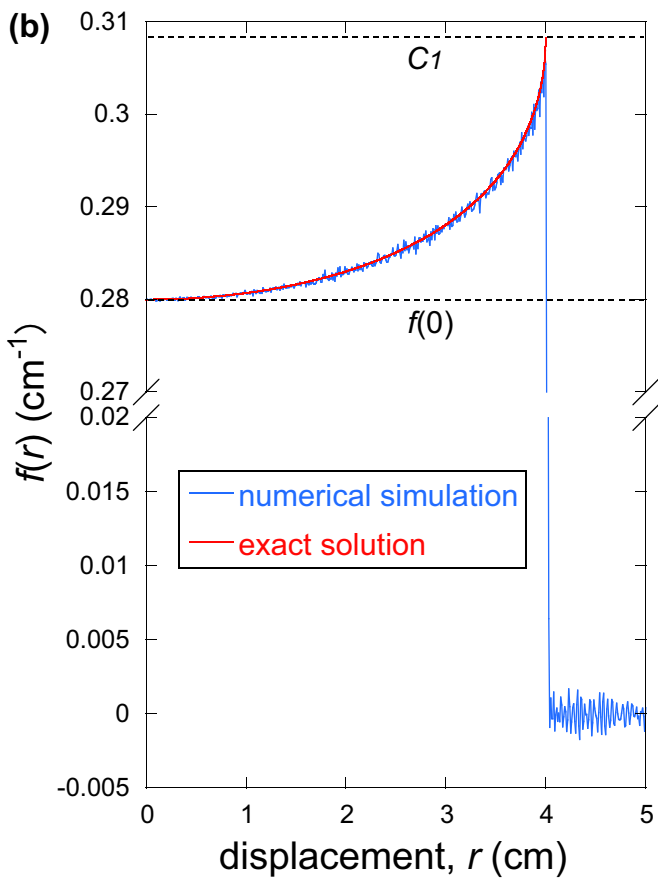

ple center from the sample rim) can be observed. The random fluctuations in the voxel value throughout the profile are derived from the discretization of the cylindrical sample shape using finite voxel dimensions of $0.01^{2} \mathrm{~cm}^{2}$. The exact solution of $f(r)$ in Eq. (1), which corresponds to the infinitesimal voxel dimension, is also superimposed for $r<4 \mathrm{~cm}$ and is free from such fluctuations. Two values of $C_{1}=0.3084 \mathrm{~cm}^{-1}$ and $f(0)=0.2800 \mathrm{~cm}^{-1}$ are indicated by the dashed lines

of the X-ray tube. For example, $E_{\max }$ is $100 \mathrm{keV}=16.0 \mathrm{fJ}$ for $100 \mathrm{kV}$ and is $120 \mathrm{keV}=19.2 \mathrm{fJ}$ for $120 \mathrm{kV}$. The coefficient $C_{n}$ for $n \geq 2$ can be readily calculated using a simple recurrence relation with the $n$th moment of the LAC spectrum of the sample [19].

The continuous distribution $f(r)$ can be reasonably characterized using only two values, namely the reconstructed voxel values at the center [i.e. $f(0)]$ and in the vicinity of the rim of the cylindrical sample [9]. Based on Eq. (1), the limiting behavior at the sample rim [19] is that $f(r) \rightarrow C_{1}$ as $r \rightarrow R$. Therefore, it is reasonable to assume that $f(r) \approx$ $C_{1}$ in the immediate vicinity of the sample rim (Fig. 2b). The values of $f(0)$ and $C_{1}$ are thus utilized as measures to characterize the severity of cupping artifacts.

We consider the following simple object as a sample. A simple substance containing a heavy element and pure water is confined in a cylindrical plastic container. The $\mathrm{X}$-ray absorption of the very thin plastic container is negligible compared to that of the heavy element. The sample is a homogeneous aqueous solution or fine-grained suspension, meaning the ion/grain size of the simple substance 
is very small compared to the voxel dimension and it can be accurately approximated as a homogeneous substance.

The effects of sample chemistry and acceleration voltage on the value of $C_{1}$ value were calculated systematically for a fixed value of $f(0)$ according to the procedure presented by Nakano and Nakashima [19]. The detailed input data for the exact solutions were as follows. Heavy elements ranging from ${ }_{47} \mathrm{Ag}$ to ${ }_{84} \mathrm{Po}$ were examined as simple substances. The radius of the sample $R$ was $4 \mathrm{~cm}$, and $N$ was 100 . X-ray source spectra were derived for acceleration voltages of 100 and $120 \mathrm{kV}$ (Fig. 1). The value of $f(0)$ was tentatively fixed at $0.2800\left(\approx 200\right.$ Hounsfield units (HU)) and $0.2571 \mathrm{~cm}^{-1}$ for the acceleration voltages of 100 and $120 \mathrm{kV}$, respectively. These parameters correspond to an $\mathrm{I}-\mathrm{H}_{2} \mathrm{O}$ mixture $(43.0 \mathrm{mM}$ iodine). We followed the method presented by Nakashima and Nakano $[9,10]$ to estimate the bulk density of the mixture, which was required for LAC spectrum calculations based on the National Institute of Standards and Technology database [21]. Three examples of calculated LAC spectra are presented in Fig. 1.

For the iodine-bearing samples, in addition to the line profile for the $\mathrm{I}-\mathrm{H}_{2} \mathrm{O}$ mixture, a line profile for a $\mathrm{KI}-\mathrm{H}_{2} \mathrm{O}$ mixture (42.2 mM KI) was also calculated. The reconstructed voxel values of the CT images are very sensitive to the atomic number of the heaviest element in the sample and less sensitive to the atomic numbers of lighter elements $[12,22]$. To demonstrate this lesser sensitivity, an iodine compound containing a lighter element (i.e., potassium) was considered. A 42.2-mM KI solution was considered because the corresponding concentration value yields the same $f(0)$ value (i.e., $0.2800 \mathrm{~cm}^{-1}$ ) for an acceleration voltage of $100 \mathrm{kV}$.

\section{Results}

Figure $2 b$ presents a representative example of the theoretical cupping profile of the $\mathrm{I}_{-} \mathrm{H}_{2} \mathrm{O}$ mixture in Fig. 1 (43.0$\mathrm{mM}$ iodine mixture). The theoretical curve $f(r)$ is relatively smooth compared to the noisy line profile obtained numerically by using an image-reconstruction computer program. Therefore, the use of the exact solution from Eq. (1) ensures that the severity of cupping artifacts can be assessed with high accuracy.

As for the 38 heavy elements ranging from ${ }_{47} \mathrm{Ag}$ to ${ }_{84} \mathrm{Po}$, the values of $C_{1}$ and the molar concentrations determined using Eq. (1) are plotted in Fig. 3 against the atomic numbers of each heavy element in the aqueous mixture. The data points in Fig. 3 were determined by a trial-anderror search for the $C_{1}$ value and molar concentration of each heavy element that most reasonably reproduced the $f(0)$ value of $0.2800 \mathrm{~cm}^{-1}$ for $100 \mathrm{kV}$. The trial-anderror search was also performed for the $f(0)$ value of

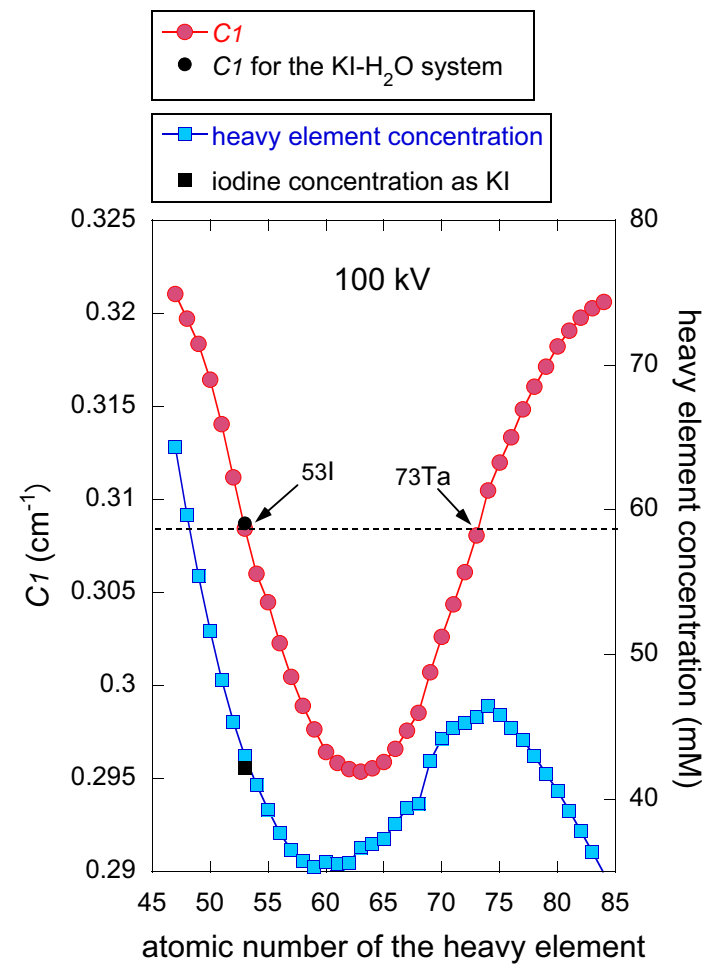

Fig. 3 Dependence of the $C_{1}$ value on the atomic number of the heavy element in the aqueous sample for a fixed $f(0)$ value of $0.2800 \mathrm{~cm}^{-1}$. The X-ray source spectrum for an acceleration voltage of $100 \mathrm{kV}$ (Fig. 1) is considered. The $C_{1}$ value for the $\mathrm{I}_{-} \mathrm{H}_{2} \mathrm{O}$ mixture is indicated by the dashed line. The molar concentrations of the heavy elements corresponding to each $C_{1}$ value are also plotted. Data points for the $\mathrm{KI}-\mathrm{H}_{2} \mathrm{O}$ mixture ( $42.2 \mathrm{mM}$ of potassium iodide) are superimposed, revealing a reasonable agreement with those for the $\mathrm{I}-\mathrm{H}_{2} \mathrm{O}$ system (43.0 $\mathrm{mM}$ of iodine)

$0.2571 \mathrm{~cm}^{-1}$ with respect to $120 \mathrm{kV}$; the results are plotted in Fig. 4, showing $C_{1}$ values and molar concentrations significantly different from those in Fig. 3. The results for the three samples whose LAC spectra were presented in Fig. 1 are also included in Figs. 3 and 4. The characteristic $C_{1}$ curves with minimum values in Figs. 3 and 4 are essentially identical to the curve presented in the study by Nakashima and Nakano [9] (see Fig. 6 in the literature). The curves with minimum values are a consequence of the sensitivity of the severity of cupping artifacts to the relative position between the $\mathrm{K}$ absorption edge of a heavy element and the spectral peak of an X-ray source $[9,16$, 17].

All data needed for the material differentiation using double-exposure CT are given in Figs. 3 and 4. The data should be arranged for convenience in terms of the determination of the atomic number and molar concentration. Thus, the data points in Figs. 3 and 4 were re-plotted as cross-plots of the $C_{1}$ values in Fig. 5 and of the molar concentrations in Fig. 6. The trajectories depicted in Figs. 5 and 6 as functions of the atomic numbers of the heavy elements in the cylindrical 




Fig. 4 This figure is the same as Fig. 3, except that the X-ray source spectrum corresponds to an acceleration voltage of $120 \mathrm{kV}$ (see Fig. 1). The $f(0)$ value was fixed at $0.2571 \mathrm{~cm}^{-1}$

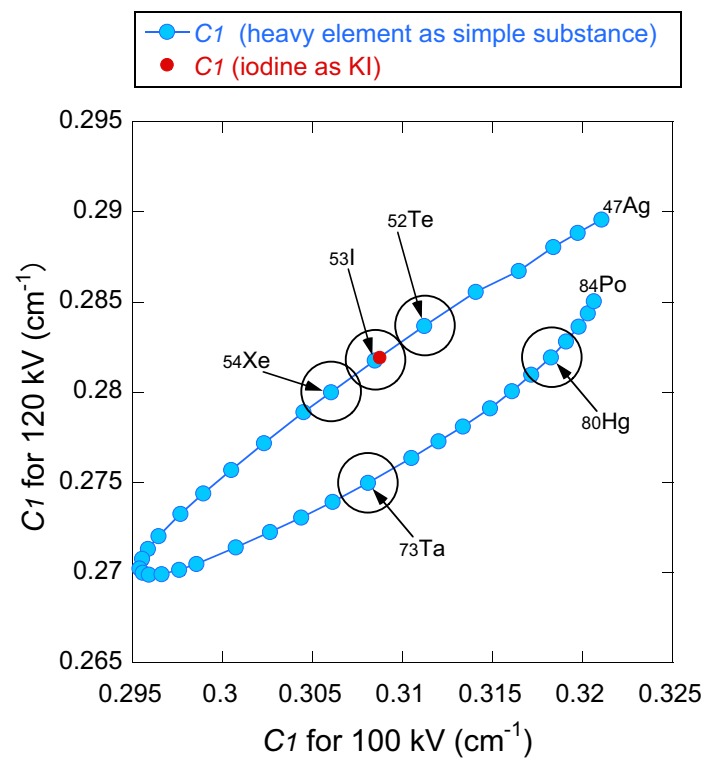

Fig. 5 Cross-plot of the $C_{1}$ values for acceleration voltages of 100 and $120 \mathrm{kV}$. The data came from Figs. 3 and 4. Tentative errors for an actual CT system are indicated by the five circles centered at ${ }_{52} \mathrm{Te},{ }_{53} \mathrm{I}$, ${ }_{54} \mathrm{Xe},{ }_{73} \mathrm{Ta}$, and ${ }_{80} \mathrm{Hg}$. The radii of the circles are $0.0016 \mathrm{~cm}^{-1}$. Note the overlap of the circles for ${ }_{53} \mathrm{I}$ and ${ }_{54} \mathrm{Xe}$

container are important for the process of heavy element quantification, as discussed in the following section.

In contrast to the exact solutions derived from Eq. (1) in this study, experimentally obtained CT images acquired using a CT apparatus contain undesirable artifacts [23] and

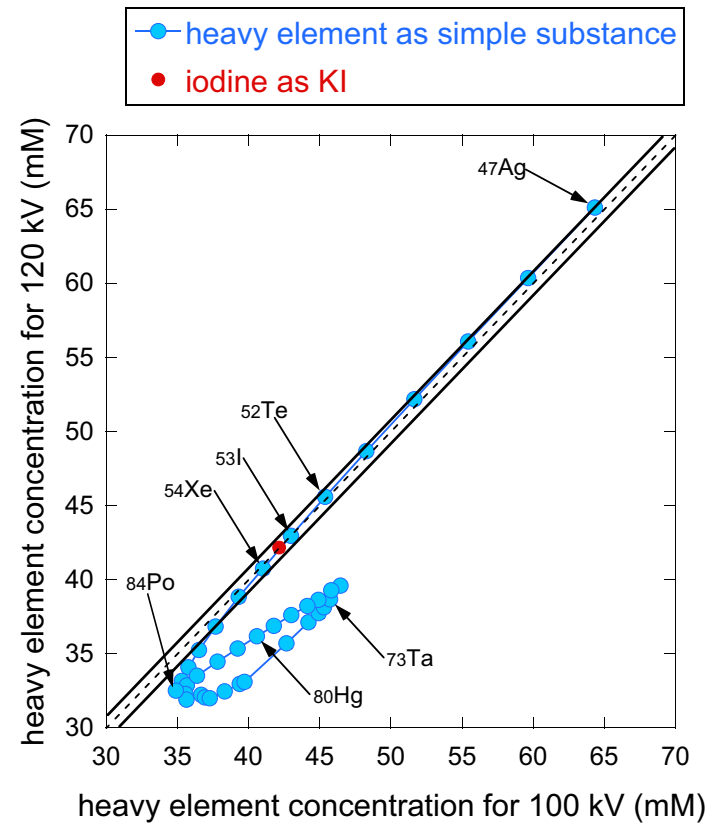

Fig. 6 Cross-plot of the heavy element concentrations for acceleration voltages of 100 and $120 \mathrm{kV}$. The data came from Figs. 3 and 4 . Exact agreement is indicated by the dashed line. Tentative errors for an actual CT system are indicated by the solid lines $( \pm 0.8 \mathrm{mM})$

noise [24]. Measurement errors caused by the noise and artifacts may reduce the accuracy of the determination of atomic numbers and concentrations of heavy elements [9]. Based on the literature [24] on the experimentally obtained standard deviation of the voxel-value fluctuation for real CT imagery, an error level of $7 \mathrm{HU}$ corresponding to $0.0016 \mathrm{~cm}^{-1}$ was tentatively chosen in the present study. The error level of $0.0016 \mathrm{~cm}^{-1}$ was expressed as the radii of circles in Fig. 5 . The dataset in Fig. 3 implies that fluctuation in $f$ values $\left( \pm 0.0016 \mathrm{~cm}^{-1}\right)$ results in fluctuation of molar concentrations of approximately $\pm 0.8 \mathrm{mM}$, which is indicated by solid lines in Fig. 6.

\section{Discussion}

The quantification of a heavy element in a sample based on the analysis of double-exposure CT images can be performed as follows. Iodine that is one of hazardous heavy elements was chosen as the target element in the present study. We assume that the ground truth is the $43.0-\mathrm{mM}$ iodinewater mixture, which yields values of $C_{1}=0.3084 \mathrm{~cm}^{-1}$ and $f(0)=0.2800 \mathrm{~cm}^{-1}$ at $100 \mathrm{kV}$, and $C_{1}=0.2818 \mathrm{~cm}^{-1}$ and $f(0)=0.2571 \mathrm{~cm}^{-1}$ at $120 \mathrm{kV}$. Therefore, the problem can be defined as using Figs. 5 and 6 to search for the atomic number and molar concentration of the heavy element in the cylindrical aqueous sample with $R=4 \mathrm{~cm}$ that reproduce the values of $C_{1}$ and $f(0)$ mentioned above. 
Although the LAC spectra are very different between the 45.7-mM tantalum and $43.0 \mathrm{mM}$-iodine aqueous samples (see Fig. 1), the sample containing ${ }_{73}$ Ta yields nearly the same $C_{1}$ value (i.e., $0.3081 \mathrm{~cm}^{-1}$ ) as the sample containing ${ }_{53} \mathrm{I}$ (i.e., $0.3084 \mathrm{~cm}^{-1}$ ) at $100 \mathrm{kV}$ (Fig. 3). This makes it difficult to eliminate ${ }_{73} \mathrm{Ta}$ from the heavy element candidates for the sample. It is a poor result to fail to distinguish iodine from tantalum because their atomic numbers are very different. A similar difficulty arises at $120 \mathrm{kV}$ (Fig. 4) for the $C_{1}$ value of ${ }_{80} \mathrm{Hg}$ (i.e., $0.2819 \mathrm{~cm}^{-1}$ ), which is nearly the same as that of ${ }_{53} \mathrm{I}$ (i.e., $0.2818 \mathrm{~cm}^{-1}$ ). This difficulty was encountered in a previous study [9] on heavy element quantification using single-exposure CT.

This difficulty can be avoided by using double-exposure CT based on Fig. 5. An error circle centered on the iodinewater mixture is drawn in Fig. 5 to represent the ground truth. The circle overlaps only with the adjacent circle centered on the xenon-water mixture. No overlap occurs with the circles for tellurium, tantalum, and mercury. Therefore, the heavy elements that reproduce the values of $C_{1}$ and $f(0)$ are iodine and xenon. It may be acceptable to fail to eliminate ${ }_{54} \mathrm{Xe}$ as a candidate because its LAC spectrum is nearly the same as the ${ }_{53}$ I spectrum (see Fig. 1). It should be noted that double-exposure CT enables the successful elimination of tantalum and mercury, while single-exposure CT fails to eliminate these candidates. This is a physical consequence of $C_{1}$ being sensitive to the acceleration voltage of the X-ray tube for a fixed value of $f(0)$, which allows it to provide the different constraints required for element identification based on $\mathrm{CT}$ at different acceleration voltages.

Once the atomic numbers of the candidates are determined, the molar concentrations can be readily estimated based on Fig. 6. According to Fig. 6, the iodine concentration is $43.0 \mathrm{mM}$ at both 100 and $120 \mathrm{kV}$, whereas the xenon concentration is $41.0 \mathrm{mM}$ at $100 \mathrm{kV}$ and $40.8 \mathrm{mM}$ at $120 \mathrm{kV}$. The two data points for iodine and xenon in Fig. 6 fall within the error lines of $\pm 0.8 \mathrm{mM}$, which confirms that the choice of these two elements is self-consistent and reasonable. It also should be noted that tantalum and mercury are located out of the error lines of $\pm 0.8 \mathrm{mM}$, demonstrating that the two candidates are eliminated again in Fig. 6.

The data points for the $\mathrm{KI}-\mathrm{H}_{2} \mathrm{O}$ mixture $(42.2 \mathrm{mM}$ potassium iodide) agree well with those for the $\mathrm{I}-\mathrm{H}_{2} \mathrm{O}$ mixture (43.0 mM iodine). This is because $C_{1}$ values are less sensitive to the atomic numbers of lighter elements, such as ${ }_{19} \mathrm{~K}$, while they are more sensitive to those of the heaviest elements [22]. This ensures that the accuracy of the doubleexposure CT method is not significantly affected by species of light elements in compounds with heavy elements.

Figures 5 and 6 demonstrate the advantage of the present study over the conventional double-exposure CT method. In the conventional method, $f(r)$ is fixed to be almost constant within the homogeneous cylindrical sample (i.e., $f(0) \approx C_{1}$ ) because the cupping artifact is suppressed by specific methods $[8,13-15,20]$. As a result, Fig. 5 is no longer available, and only the constraint on self-consistency (Fig. 6) can be used for the heavy-element determination. According to Fig. 6, as many as ten candidates $\left({ }_{47} \mathrm{Ag}\right.$ to $\left.{ }_{56} \mathrm{Ba}\right)$ fall within the error lines of $\pm 0.8 \mathrm{mM}$. In contrast, the combination of Figs. 5 and 6 allows only ${ }_{53} \mathrm{I}$ and ${ }_{54} \mathrm{Xe}$ as candidates, demonstrating the advantage of the method of the present study.

The method for CT-based heavy element quantification that was proposed by Nakashima and Nakano $[9,10]$ is unique in that it utilizes the dependence of the atomic number and concentration on the cupping profile. The accuracy of this method can be improved by employing doubleexposure CT, as shown in Figs. 5 and 6. This is analogous to the advantages of two-dimensional nuclear magnetic resonance (NMR) [25] compared to one-dimensional NMR, where using two dimensions significantly improves the ability to differentiate materials. However, a careful comparison between the image analysis methods proposed in this paper and the conventional double-exposure X-ray imagery [7, 26-29] should be performed in future. Additional directions for future research include the following four subjects.

(i) We have demonstrated successful heavy element identification using the analytical solution in Eq. (1) for a cylindrical phantom sample. One of the next possible research steps is to analyze real samples (e.g., natural ores or polluted soils with heavy elements). However, the shape of the real samples is not always cylindrical. The applicability of the proposed method to a sample with an irregular shape (noncylindrical shape) should be evaluated. Numerical (not analytical) image reconstruction simulations [9, 10] for reproducing experimentally obtained beam hardening could be a possible solution.

(ii) Real samples often contain two or more heavy elements as compounds (e.g., $\mathrm{Hg}_{2} \mathrm{I}_{2}$ ). While Figs. 3, 4, 5,6 show that effects of a light element in a compound (i.e., potassium in potassium iodide) are negligible, those of heavy elements (e.g., mercury in mercury iodide) would be significant due to the strong X-ray attenuation. Thus, re-calculation of the all curves in Figs. 3, 4, 5, 6 is needed when applying the method of the present study to compounds with two or more heavy elements.

(iii) Regarding lanthanoids, the first derivative of the $C_{1}$ curve is nearly zero and the $C_{1}$ value calculated using Eq. (2) is less sensitive to the atomic number (see the minimum values in Figs. 3 and 4). Therefore, if the ground truth is a lanthanoid, then estimation accuracy would decrease because many candidates are included in the error circles of $0.0016 \mathrm{~cm}^{-1}$ in Fig. 5. This problem could be solved by perform- 
ing $\mathrm{CT}$ at acceleration voltages other than 100 and $120 \mathrm{kV}$ (e.g., 80 and $140 \mathrm{kV}$ ). This is because the position of the $C_{1}$ minimum value shifts with the acceleration voltage, as confirmed by a comparison between Figs. 3 and 4. Additionally, the reduction of the radius of the error circle by increasing the tube current could be another solution.

(iv) This study is based on the physics of beam hardening that is derived from the non-linear relationship between the X-ray projection (logarithmic intensity of the attenuated X-ray) and the sample penetration depth [19]. Such non-linearity also occurs in X-ray radiography (not tomography) of a sample with an irregular shape [28]. Thus, the method of the present study could be potentially extended to the doubleexposure radiography [29], which is an economically more reasonable apparatus than tomography.

\section{Conclusions}

The principle for nondestructive heavy element identification by means of double-exposure X-ray CT was presented for analytical chemistry. This method involves quantitative CT image analysis of the beam hardening resulting from the use of a polychromatic X-ray source. By using analytical expressions for the cupping profile of the beam hardening for a homogeneous cylindrical sample, as well as the dependence of the profile on the atomic number and concentration of a heavy element, and on the acceleration voltage of the X-ray tube, heavy elements can be systematically identified. Careful analysis of these dependencies enabled the atomic numbers and molar concentrations of heavy elements to be determined simultaneously within a certain estimation error range.

Acknowledgements Comments by three anonymous reviewers were helpful. The ImageJ program developed by the National Institutes of Health was used for the line profile analysis of CT image in Fig. 2. Preliminary CT experiments on cylindrical samples were performed at the AIST GSJ-Lab, Japan Synchrotron Radiation Research Institute (Proposal no. 2001B0501-NOD-np), and Center for Advanced Marine Core Research (CMCR) at Kochi University (13B034) with support from JAMSTEC. This study was supported in part by a KAKENHI Grant-in-Aid (No. 23241012) from the Japan Society for the Promotion of Science (JSPS).

\section{Compliance with Ethical Standards}

Conflict of interest The authors are inventors of a Japanese patent (Patent Number P5946095) of which contents are related to the references $[9,10]$.

Open Access This article is licensed under a Creative Commons Attribution 4.0 International License, which permits use, sharing, adaptation, distribution and reproduction in any medium or format, as long as you give appropriate credit to the original author(s) and the source, provide a link to the Creative Commons licence, and indicate if changes were made. The images or other third party material in this article are included in the article's Creative Commons licence, unless indicated otherwise in a credit line to the material. If material is not included in the article's Creative Commons licence and your intended use is not permitted by statutory regulation or exceeds the permitted use, you will need to obtain permission directly from the copyright holder. To view a copy of this licence, visit http://creativecommons org/licenses/by/4.0/.

\section{References}

1. Vanhoof C, Bacon JR, Ellis AT, Fittschen UE, Vincze L (2019) 2019 atomic spectrometry update-a review of advances in X-ray fluorescence spectrometry and its special applications. J Anal At Spectrom 34:1750-1767

2. Petrozziello A, Jordanov I (2019) Automated deep learning for threat detection in luggage from X-ray images. In: International symposium on experimental algorithms. Springer, Cham, pp 505-512

3. Hilal MA, Abdelbary HM, Mohamed GG (2018) Physicochemical and radiation hazardous properties of scale TENORM waste: evaluation by different analytical techniques. Radiochemistry 60:444-449

4. Verla EN, Verla AW, Enyoh CE (2020) Bioavailability, average daily dose and risk of heavy metals in soils from children playgrounds within Owerri, Imo State, Nigeria. Chem Afr (in press)

5. Adekunle AS, Oyekunle JAO, Ojo OS, Makinde OW, Nkambule TT, Mamba BB (2020) Heavy metal speciation, microbial study and physicochemical properties of some groundwaters: a case study. Chem Afr 3:211-226

6. Guenane F, Kerchich Y, Hachemi M (2020) Heavy metals in flue gas emission and ash generated by an incineration plant for hospital and industrial waste in Northern of Algeria. Algerian J Environ Sci Technol (in press)

7. Champley KM, Azevedo SG, Seetho IM, Glenn SM, McMichael LD, Smith JA, Kallman JS, Brown WD, Martz HE (2019) Method to extract system-independent material properties from dual-energy X-ray CT. IEEE Trans Nucl Sci 66:674-686

8. Yang F, Zhang D, Zhang H, Huang K (2020) Cupping artifacts correction for polychromatic X-ray cone-beam computed tomography based on projection compensation and hardening behavior. Biomed Signal Process Control 57:101823

9. Nakashima Y, Nakano T (2012) Nondestructive quantitative analysis of a heavy element in solution or suspension by single-shot computed tomography with a polychromatic X-ray source. Anal Sci 28:1133-1138

10. Nakashima Y, Nakano T (2013) Quantitative analysis of a heavy element in a solution sample by medical X-ray CT. In: The 69th annual scientific congress of the Japanese society of radiological technology (Yokohama, Japan)

11. Hamaguchi T, Kanno I (2019) Effective atomic number estimation by energy-resolved X-ray computed tomography with a currentmode detector system. Jpn J Appl Phys 58:071001

12. Busi M, Mohan KA, Dooraghi AA, Champley KM, Martz HE, Olsen UL (2019) Method for system-independent material characterization from spectral X-ray CT. NDT \& E Int 107:102136

13. Nakano T, Nakashima Y, Nakamura K, Ikeda S (2000) Observation and analysis of internal structure of rock using X-ray CT. J Geol Soc Jpn 106:363-378 (in Japanese with English abstract) 
14. Nakashima Y (2003) Diffusivity measurement of heavy ions in Wyoming montmorillonite gels by X-ray computed tomography. J Contam Hydrol 61:147-156

15. Bismark RN, Frysch R, Abdurahman S, Beuing O, Blessing M, Rose G (2020) Reduction of beam hardening artifacts on real $\mathrm{C}$-arm CT data using polychromatic statistical image reconstruction. Z Med Phys 30:40-50

16. Nakashima Y (2013) The use of sodium polytungstate as an X-ray contrast agent to reduce the beam hardening artifact in hydrological laboratory experiments. J Hydrol Hydromech 61:347-352

17. Nakashima Y, Nakano T (2014) Optimizing contrast agents with respect to reducing beam hardening in nonmedical X-ray computed tomography experiments. J X-ray Sci Technol 22:91-103

18. Goertzen AL, Beekman FJ, Cherry SR (2002) Effect of phantom voxelization in CT simulations. Med Phys 29:492-498

19. Nakano T, Nakashima Y (2018) Analytical expressions for the reconstructed image of a homogeneous cylindrical sample exhibiting a beam hardening artifact in X-ray computed tomography. $\mathrm{J}$ X-ray Sci Technol 26:691-705

20. Yang K, Ruan C, Li X, Liu B (2019) Data of CT bow tie filter profiles from three modern CT scanners. Data Br 25:104261

21. Berger MJ, Hubbell JH, Seltzer SM, Chang J, Coursey JS, Sukumar R, Zucker DS, Olsen K (2010) XCOM: Photon Cross Sections Database (National Institute of Standards and Technology). https ://dx.doi.org/10.18434/T48G6X. Accessed 5 Mar 2020

22. Nakashima Y (2000) The use of X-ray CT to measure diffusion coefficients of heavy ions in water-saturated porous media. Eng Geol 56:11-17
23. Orhan K, de Faria Vasconcelos K, Gaêta-Araujo H (2020) Artifacts in micro-CT. In: Micro-computed tomography (micro-CT) in medicine and engineering. Springer, Cham, pp 35-48

24. Funama Y, Awai K, Hatemura M, Shimamura M, Yanaga Y, Oda S, Yamashita Y (2008) Automatic tube current modulation technique for multidetector CT: is it effective with a 64-detector CT? Radiol Phys Technol 1:33-37

25. Kovrlija R, Goubin E, Rondeau-Mouro C (2020) TD-NMR studies of starches from different botanical origins: hydrothermal and storage effects. Food Chem 308:125675

26. Osipov S, Chakhlov S, Udod V, Usachev E, Schetinkin S, Kamysheva E (2020) Estimation of the effective mass thickness and effective atomic number of the test object material by the dual energy method. Rad Phys Chem 168:108543

27. Kordbacheh H, Baliyan V, Uppot RN, Eisner BH, Sahani DV, Kambadakone AR (2019) Dual-source dual-energy CT in detection and characterization of urinary stones in patients with large body habitus: observations in a large cohort. Am J Roentgenol 212:796-801

28. Baur M, Uhlmann N, Pöschel T, Schröter M (2019) Correction of beam hardening in X-ray radiograms. Rev Sci Instrum 90:025108

29. Veras MM, Young AS, Born CR, Szewczuk A, Neto ACB, Petter CO, Sampaio CH (2020) Affinity of dual energy X-ray transmission sensors on minerals bearing heavy rare earth elements. Miner Eng 147:106151 